\title{
Third-order correlations in cavity quantum electrodynamics
}

\author{
G T Foster ${ }^{1}$, W P Smith, J E Reiner and L A Orozco \\ Department of Physics and Astronomy, State University of New York at Stony Brook, \\ Stony Brook, NY 11794-3800, USA \\ E-mail: luis.orozco@sunysb.edu
}

Received 18 December 2001

Published 29 July 2002

Online at stacks.iop.org/JOptB/4/S281

\begin{abstract}
The correlation of a photon detection with the record of a homodyne photocurrent has allowed the observation of time-resolved quantum fluctuations of the amplitude of a squeezed electromagnetic wave in a cavity quantum electrodynamic system. The photon detection, through the wavefunction collapse, prepares a conditional state whose evolution back to equilibrium is observed. The perturbation of the wave amplitude is measured at the sub-photon level. The third-order correlation is manifestly non-classical, giving a minimum amplitude at zero delay where there would classically be a maximum; its Fourier transform gives the squeezing spectrum.
\end{abstract}

Keywords: Non-classical light, quantum noise, cavity QED, squeezing, antibunching

\section{Introduction}

The nature of light is a topic that has animated discussions in physics since the time of Isaac Newton. The argument of wave versus particle is resolved in quantum electrodynamics (QED) by a formalism that combines both of these aspects. The formalism is fundamentally statistical, and as with quantum phenomena in general, it is through the statistical uncertaintyfluctuations-that the wave and particle natures of light sit self-consistently side by side.

Following from the development of the laser, quantum optics has made a detailed study of the quantum fluctuations of light. Two lines of experiments are of particular interest: those measuring correlations between pairs of photon detections (particle aspect of light) [1-5]; and squeezing experiments, which measure the fluctuation variance of the wave amplitude of light [6-8]. No attempt has been made previously to draw on the particle and wave aspects together by correlating a photon detection with fluctuations of the electromagnetic wave amplitude. We have managed this, using a cavity QED system as a source, where a single mode of the electromagnetic field interacts with a collection of two-state atoms [9]. The

\footnotetext{
1 Present address: Department of Physics, Yale University, New Haven, CT 06520-8120, USA
}

source is known to emit non-classical light; this has been demonstrated in both photon correlation [3-5] and squeezing measurements [10-12]. We observe the fluctuation of the wave amplitude of light underlying these measurements [13,14] by exploiting the conditioning on a photon detection to catch the fluctuation as it occurs. The fluctuation is measured in its evolution over time in an efficiency-independent manner, from which the full spectrum of squeezing is obtained. We achieve this by measuring the electric field of a fraction of a photon, a signal smaller than the shot noise by two orders of magnitude. Squeezing is measured conventionally in the frequency domain using a non-conditional technique that suffers from detection efficiency loss.

In this contribution to the Proceedings of Garda 2001, we present a brief review of our recent work on third-order correlations of the electromagnetic field. We apply it to our optical cavity QED system $[15,16]$. We begin with a review of the third-order (wave-particle) correlation function. We then look at the correlation in cavity QED. We present our experimental realization with results and finish with a short conclusion.

\section{Wave-particle correlation function}

Correlation functions are an ideal tool for characterizing the electromagnetic field. Most correlation measurements 


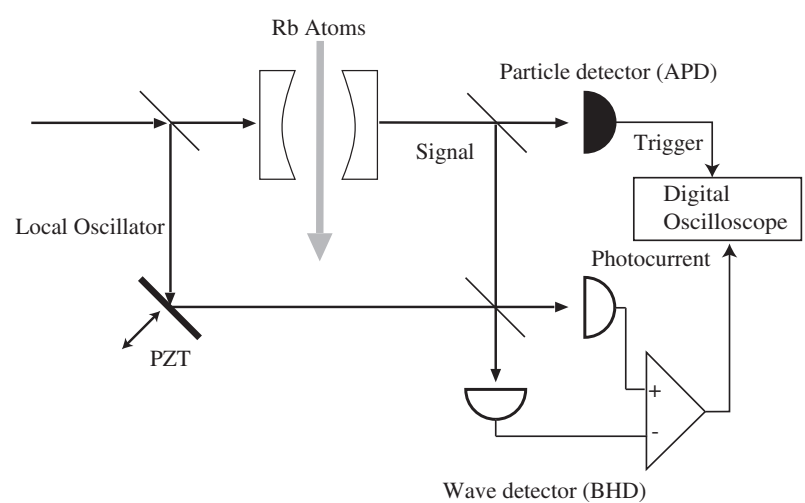

Figure 1. Simplified diagram of the third-order correlator in our cavity QED experimental set-up.

emphasize even-order functions of the field (see, for example, [17]). We work with a third-order correlation function $[15,16]$ of an electromagnetic field mode $\hat{b}$ (signal) that has a non-zero, steady-state average $\langle\hat{b}\rangle=\lambda$. Carmichael et al [15] have generalized it to any source.

The apparatus (see figure 1) measures the correlation between the amplitude and the intensity of the electromagnetic field. The particle produces a trigger 'click' and the wave is recorded in the photocurrent output of a balanced homodyne detector (BHD). The measured correlation is

$$
\left\langle:\left(\hat{b}^{\dagger} \hat{b}\right)(0) \hat{Q}_{\theta}(\tau):\right\rangle=\left\langle:\left(\hat{Q}_{\theta}(\tau):\right\rangle_{c},\right.
$$

where $\hat{Q}_{\theta} \equiv \frac{1}{2}\left(\hat{b} \mathrm{e}^{-\mathrm{i} \theta}+\hat{b}^{\dagger} \mathrm{e}^{\mathrm{i} \theta}\right)$ is the quadrature amplitude selected by the local oscillator (LO) phase $\theta$. The colons indicate normal ordering and the operator is time ordered. Equation (1) represents the measurement of the field after a photon has been detected. Using the quantum regression theorem (see, for example, a similar treatment for the secondorder intensity correlation in [14]), we write the correlation function, denoted by the subscript $c$, as the field evolution conditioned to detection of one photon.

We normalize equation (1) by $\sqrt{\eta}|\langle\hat{b}\rangle|\left\langle\hat{b}^{\dagger} \hat{b}\right\rangle$, where $\eta$ is the coupling efficiency into the BHD, and we separate the fluctuations from the mean field by writing $\hat{b}=|\langle\hat{b}\rangle| \mathrm{e}^{\mathrm{i} \theta}+\Delta \hat{b}$. When the mean field has the same phase as the LO and for Gaussian fluctuations (third-order moments vanish) the correlation function is

$$
h_{\theta}(\tau)=\cos \theta+2 \frac{\left\langle: \Delta \hat{Q}_{\theta}(0) \Delta \hat{Q}_{\theta}(\tau):\right\rangle}{\lambda^{2}+\left\langle\Delta \hat{b}^{\dagger} \Delta \hat{b}\right\rangle}+\xi(\tau),
$$

where $\Delta \hat{Q}_{\theta} \equiv \frac{1}{2}\left(\Delta \hat{b} \mathrm{e}^{-\mathrm{i} \theta}+\Delta \hat{b}^{\dagger} \mathrm{e}^{\mathrm{i} \theta}\right)$ and the residual shot noise $\xi(\tau)$ obeys the following correlation function

$$
\overline{\xi(0) \xi(\tau)}=\frac{\Gamma}{16 \eta N_{s}|\langle\hat{b}\rangle|^{2}} \mathrm{e}^{-\Gamma \tau}
$$

where $\Gamma$ is the BHD bandwidth and $N_{s}$ is the number of triggers the correlator has received from photon detections. The strength of the LO is irrelevant; it cancels out of this correlation function and it only enters in the shot noise that should be averaged out to obtain a good signal-to-noise ratio.

A connection to non-classicality follows since the spectrum of squeezing is the cosine transform of the normalized correlation function [15]

$$
S(\theta, \nu)=4 F \int_{0}^{\infty} \mathrm{d} \tau \cos (2 \pi \nu \tau)\left[\overline{h_{\theta}(\tau)}-1\right],
$$

where $F=2 \kappa\left\langle\hat{b}^{\dagger} \hat{b}\right\rangle$ is the photon flux into the correlator and $\overline{h_{\theta}(\tau)}$ is the average of $h_{\theta}(\tau)$ with respect to $\xi(\tau)$ taken with many realizations, $N_{s}$, of the measurement. This average is independent of the fraction of light sent to the BHD.

Notice that the measured correlation function equation (2) depends on the BHD efficiency, $\eta$, only in the residual shot noise term, which vanishes after averaging. The conditional measurement avoids the degradation of squeezing encountered by the standard squeezing measurement.

A related conditional detection scheme has been proposed and implemented for observing photon number states in parametric down-conversion $[18,19]$. That scheme does not display the efficiency independence observed here.

The signature of squeezing is a reduction in the shot noise level which is interpreted as a de-amplification of the vacuum fluctuations. The wave-particle correlation function brings another interpretation. The focus is on the fluctuations of the light emitted by the source. The shot noise is irrelevant, ideally being eliminated through the conditional average. The fluctuation intensity can be written in terms of the individual quadrature variances

$$
\left\langle\Delta \hat{b}^{\dagger} \Delta \hat{b}\right\rangle=\left\langle:\left(\Delta \hat{Q}_{\theta}\right)^{2}:\right\rangle+\left\langle:\left(\Delta \hat{Q}_{\theta+\pi / 2}\right)^{2}:\right\rangle .
$$

Classically, the quadrature variances are positive. Equations (2) and (5) give rise to the following Schwarz inequalities

$$
\begin{aligned}
& 0 \leqslant \overline{h_{0^{\circ}}(0)}-1 \leqslant \frac{2}{1+\lambda^{2} /\left\langle\Delta \hat{b}^{\dagger} \Delta \hat{b}\right\rangle}, \\
& \left|\overline{h_{0^{\circ}}(\tau)}-1\right| \leqslant\left|\overline{h_{0^{\circ}(0)}}-1\right| .
\end{aligned}
$$

One quadrature variance is negative, however, if there is squeezing below the quantum limit, then the squeezed (unsqueezed) fluctuations violate the lower (upper) bound of (6), and fluctuations of either quadrature amplitude may violate (7).

Quantum mechanics accommodates this behaviour through the reduction of the wavefunction, where triggering on a photon detection collapses the equilibrium state of the source into a conditional state which evolves back to equilibrium in a characteristic way [13]. The size of this collapse depends on the strength of the nonlinearity of the source. The source must be able to deliver more than one excitation during its characteristic fluctuation time to measure the wave-particle correlation.

\section{Cavity QED system}

We apply the approach outlined above to measure the field fluctuations in the light emitted from our cavity QED system. The system is in a regime where the coherent atom-field coupling $g$ (single photon Rabi frequency) is larger than both the atomic $\gamma$ (for purely radiative decay) and cavity decay rates $\kappa$. The saturation photon number $n_{0}=4 \gamma^{2} / 3 g^{2}$ for our system is less than one tenth of a photon. This effectively limits the mean field in the cavity to less than a single photon. Quantum fluctuations become important when the mean photon number 
in a field is very low. The single atom cooperativity $C_{1}=$ $g^{2} / \kappa \gamma$ is more than one. We operate with an atomic beam which we model with $N$ maximally coupled atoms.

In the weak field limit, which assumes up to two excitations in the system, the conditional field measurement is $[13,20]$ :

$$
h_{\theta}(\tau)=(1+\mathcal{A F}(\tau)) \cos \theta .
$$

$\mathcal{F}$ has a damped oscillation at the vacuum Rabi frequency, $\mathcal{F}(\tau)=\exp (-\beta \tau)\left[\cos \left(\Omega_{0} \tau\right)+\left(\beta / \Omega_{0}\right) \sin \left(\Omega_{0} \tau\right)\right]$, with $\Omega_{0}=$ $\sqrt{g^{2} N-((\kappa-(\gamma / 2)) / 2)^{2}}$ and a decay rate of $\beta=(\kappa+$ $(\gamma / 2)) / 2 . \quad \mathcal{A}$ is the relative change of the field inside the cavity caused by the detection of a photon, $\mathcal{A}=-4 C_{1}^{2} N /[(1+$ $\left.(\gamma / 2) / \kappa)\left(1+2 C_{1} N\right)-2 C_{1}\right]$.

We are not sensitive to in-quadrature fluctuations because, on resonance, the mean field is zero at $\theta=90^{\circ}$. One possible way to get to the in-quadrature component of the fluctuations is by adding a coherent offset to the signal before sending it to the start detector and homodyne detector to provide a mean field to allow triggers at that phase [15].

Outside the weak field limit, saturation effects start to decouple the atoms and the field $[4,21]$. The intensity correlation measurements demonstrate that the non-classical features disappear at high driving intensities [5]. We have shown that the conditional field measurement can extract the non-classical coherent field evolution even when the system is at high intensity and is characterized by a classical intensity correlation [16].

\section{Experimental implementation}

Our cavity QED apparatus, described in detail in [5], consists of four key components: the cavity, the atomic beam, an excitation laser and the detector system. We drive the cavity system from one side, and observe the light transmitted out from the other. The three rates that characterize our cavity QED system are $(g, \kappa, \gamma) / 2 \pi=(12,8,6) \mathrm{MHz}$.

The optical cavity is formed by two high reflectivity curved mirrors with finesse around 23000 . The cavity is 'one sided' with input transmission mirror $10 \mathrm{ppm}$ and output transmission mirror $285 \mathrm{ppm}$. Piezoelectric transducers (PZTs) attached to the mirror holders allow us to control, through feedback, the mirror spacing.

An effusive oven produces a thermal beam of $\mathrm{Rb}$ atoms in a vacuum chamber. Final collimation is provided by a slit in the cavity holder. A liquid-nitrogen cooled $\mathrm{Cu}$ sleeve surrounds the cavity to reduce background atomic vapour.

A modified Coherent 899-01 titanium sapphire (Ti:sapphire) laser provides light for the signal beam and auxiliary beams for laser frequency locking, cavity locking and optical pumping. The signal and lock beams are on resonance with the $5 \mathrm{~S}_{1 / 2}, F=3, m_{F}=3 \rightarrow 5 \mathrm{P}_{3 / 2}, F=4, m_{F}=4$ transition of ${ }^{85} \mathrm{Rb}$ at $780 \mathrm{~nm}$. The region around the cavity has a $2.5 \mathrm{G}$ uniform magnetic field applied along the axis of the cavity. An optical pumping beam intersects the atomic beam $1 \mathrm{~cm}$ before the atoms enter the cavity and takes all the ${ }^{85} \mathrm{Rb}$ atoms of the $F=3$ level into the ground-state magnetic sublevel $F=3, m_{F}=3$.

An electro-optic modulator generates FM sidebands on the lock beam. The reflected lock beam is used to hold the cavity on resonance. During data collection, we send the beam through a chopper wheel which alternately passes the lock beam and opens the path from the cavity to the detectors at about $1.1 \mathrm{kHz}$. The lock and signal beams have orthogonal circular polarizations before the cavity. The circular polarization of the signal beam is in the same sense as the optical pumping beam. On the output side of the cavity, the beam passes through a $\lambda / 4$ plate before a polarizing beamsplitter sends the lock beam to a detector. The signal beam is directed to the wave-particle correlator.

Figure 1 shows our wave-particle correlator. Light of wavelength $780 \mathrm{~nm}$ from the Ti:sapphire laser enters a MachZehnder interferometer, driving the cavity QED system in one arm and providing an LO for the BHD in the other. The fluctuations of the signal field are detected with the BHD that has strong common mode rejection. Approximately $85 \%$ of the signal is mixed with the LO, whose relative phase we control with a PZT, and detect with the fast photodiodes (EOT2030) of the BHD. The photocurrent is ac amplified by $65 \mathrm{~dB}, 70 \mathrm{MHz}$ low pass filtered, and sampled with a fast digital oscilloscope at 2 Giga-samples/s (LeCroy 9354A). The remaining 15\% of the signal is directed to an avalanche photodiode (APD). Photon detections at the APD trigger the digital oscilloscope to record the BHD photocurrent. We lock the relative phase between the signal and LO by feeding an electrical signal to a PZT in the Mach-Zehnder. To derive the error for this feedback loop, we use the interference fringes from either a diode laser locked with FM sidebands to an $I_{2}$ resonance around $640 \mathrm{~nm}$, or a $\mathrm{HeNe}$ laser at $632 \mathrm{~nm}$.

The averaging and processing is done in the oscilloscope. It takes about $5 \mathrm{~min}$ of data collection to acquire 60000 averages. The maximum number of averages is limited by the 8 bit digitizer of the oscilloscope. If the processing of the information could be done faster, we would be able to acquire a correlation much faster, since the rate of 'clicks' is tens of $\mathrm{kHz}$.

\section{Results}

Figure 2 shows our results for the conditionally averaged homodyne current $\left[h_{\theta}(\tau)\right]$ in a region of moderate field. We show results for in-phase $(a)$ and out-of-phase $(b)$ settings of the LO, and we demonstrate that the signal becomes inverted under this change of phase. The change of phase is not exactly $180^{\circ}$ because we flip the phase of the LO using the maximum or minimum of the fringe at the reference laser $(632 \mathrm{~nm})$ producing a change of only $146^{\circ}$. The oscillation at the vacuum Rabi frequency is clearly present, revealing the dynamical exchange of excitation between the cavity mode and the atoms. At the in-phase setting of the LO, the perturbed wave amplitude is minimum at $\tau=0$. Instead of random fluctuations of a classical wave amplitude, we see explicit evidence of the preparation, due to the photon detection, of a conditional quantum state with increased polarization amplitude out of phase with the intracavity field $[13,14]$.

The spectrum of squeezing calculated from equation (4) is plotted in figure 3 from the data presented in figure $2(a)$. Since there is residual shot noise in the measurement, we symmetrize $h_{0^{\circ}}(\tau)$ before taking the Fourier transform. There is a reduction of the noise below the standard quantum limit around $38 \mathrm{MHz}$. 


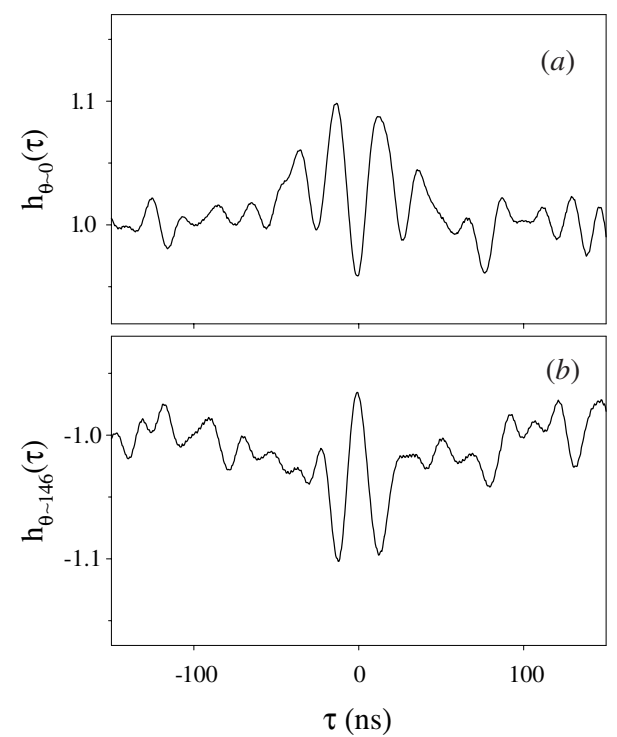

Figure 2. Normalized field correlation for in-phase $(a)$ and out-of-phase $(b)$ fields. Here $C_{1}=3, n_{0}=0.08$ and $g \sqrt{N} / 2 \pi=43 \mathrm{MHz}$, intracavity intensity $0.8 n_{0}$.

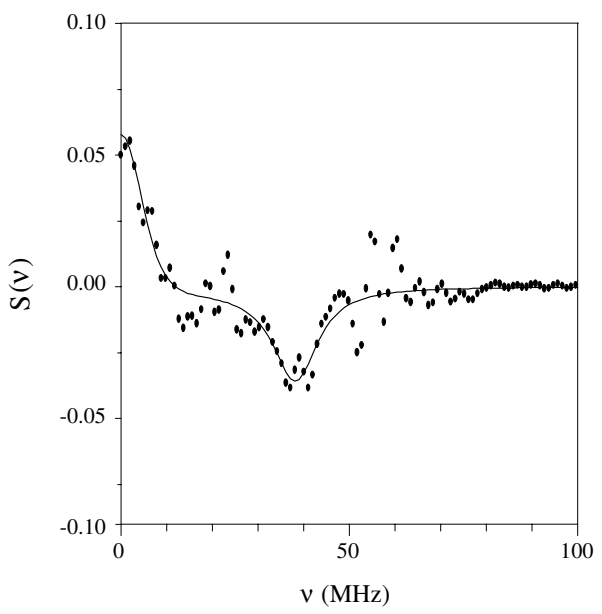

Figure 3. Squeezing spectra for cavity QED system with intracavity intensity $0.8 n_{0}$ and source flux $F=7.5 \times 10^{6}$ photons $/ \mathrm{s}$.

The squeezing is largest at a frequency slightly below the vacuum Rabi frequency $\Omega_{0}$, possibly because we are operating in the region of renormalized frequency response [21]. The system is driven on resonance and the amount of squeezing is rather small compared to previous realizations where the system was driven off resonance [10-12]. A more detailed treatment of the spectrum of squeezing in cavity QED and its relation with the correlation function $h(\tau)$ can be found in Reiner et al [20], in particular the discussion about the positive part of the spectrum at low frequency, and its relationship with spontaneous emission. The correlation function allows the time-resolved measurement of the amplitude fluctuations of the squeezed electromagnetic field. Since the normalized correlation $h(\tau)$ is independent of the detection and coupling efficiency, the values presented for the spectrum of squeezing only depend on the quantum efficiency of the photon detector used to measure the source flux.

\section{Conclusions}

We have measured the wave-particle correlation function, $h(\tau)$, in our cavity QED system. It records the conditional time evolution of the field of a fraction of a photon [16]. Detection of a photon prepares a state of well-defined phase that evolves back to equilibrium via a damped vacuum Rabi oscillation. We record the regression of the field amplitude. The correlation function is non-classical and provides an efficiency independent path to the spectrum of squeezing.

A remarkable feature of our measurement is that the detection of a photon projects the system into a well-defined quantum state, such that, from shot to shot, the fluctuation in the wave amplitude evolves in the same phased way. Thus, the fluctuation is caught reproducibly, as it occurs, which permits its recovery from the shot noise at the sub-photon level.

\section{Acknowledgments}

We thank H J Carmichael, H Castro-Beltran and P R Rice for their interest in this project. This work has been supported by the National Science Foundation and the National Institute of Standards and Technology of the USA.

\section{References}

[1] Hanbury-Brown R and Twiss R Q 1956 Nature 17727

[2] Kimble H J, Dagenais M and Mandel L 1977 Phys. Rev. Lett. 39691

[3] Rempe G, Thompson R J, Brecha R J, Lee W D and Kimble H J 1991 Phys. Rev. Lett. 671727

[4] Mielke S L, Foster G T and Orozco L A 1998 Phys. Rev. Lett. 803948

[5] Foster G T, Mielke S L and Orozco L A 2000 Phys. Rev. A 61 53821

[6] Slusher R E, Hollberg L W, Yurke B, Mertz J C and Valley J F 1985 Phys. Rev. Lett. 552409

[7] Loudon R and Knight P L (ed) 1987 Special issue on squeezed light J. Mod. Opt. 34 issues 6 and 7, pp 709-1020

[8] Kimble H J and Walls D (ed) 1987 Special issue on squeezed states of the electromagnetic field J. Opt. Soc. Am. B 4 issue 10, pp 1449-1752

[9] See, for example

Berman P (ed) 1994 Cavity Quantum Electrodynamics (Boston, MA: Academic)

[10] Raizen M G, Orozco L A, Xiao M, Boyd T L and Kimble H J 1987 Phys. Rev. Lett. 59198

[11] Orozco L A, Raizen M G, Min Xiao, Brecha R J and Kimble H J 1987 J. Opt. Soc. Am. B 41490

[12] Hope D M, Bachor H A, McClelland D E and Stevenson A 1992 Appl. Phys. B 55210

[13] Carmichael H J, Brecha R J and Rice P R 1991 Opt. Commun. 8273

[14] Brecha R J, Rice P R and Xiao M 1999 Phys. Rev. A 592392

[15] Carmichael H J, Castro Beltran H, Foster G T and Orozco L A 2000 Phys. Rev. Lett. 851855

[16] Foster G T, Orozco L A, Castro-Beltran H M and Carmichael H J 2000 Phys. Rev. Lett. 853149

[17] Mandel L and Wolf E 1995 Optical Coherence and Quantum Optics (Cambridge: Cambridge University Press)

[18] Yurke B and Stoler D 1987 Phys. Rev. A 361955

[19] Lvovsky A I, Hansen H, Aichele T, Benson O, Mlynek J and Schiller S 2001 Phys. Rev. Lett. 87050402

[20] Reiner J E, Smith W P, Orozco L A, Carmichael H J and Rice P R 2001 J. Opt. Soc. Am. B 181911

[21] Gripp J, Mielke S L and Orozco L A 1997 Phys. Rev. A 56 3262 